\title{
Supplementary material of "Detection of Saharan dust and biomass burning events using near real-time intensive aerosol optical properties in the northwestern Mediterranean"
}

5 Table S1. Statistics from the hourly averages of the considered aerosol parameters for the period under study at MSY (above) and MSA (below) sites.

\begin{tabular}{cccccccccccccc}
\hline MSY & $\lambda$ & Counts & Mean & SD & Median & Min & Max & Skewness & \multicolumn{5}{c}{ Percentiles } \\
\hline \multirow{5}{*}{$\sigma_{\text {sp }}$} & & & & & & & & & 5 & 25 & 50 & 75 & 95 \\
& 535 & 28443 & 30.05 & 27.67 & 23.53 & -0.50 & 596.55 & 3.12 & 3.01 & 11.64 & 23.53 & 40.06 & 79.46 \\
& 450 & 28522 & 38.41 & 34.03 & 30.26 & -0.50 & 539.71 & 2.52 & 4.27 & 14.98 & 30.26 & 51.72 & 100.42 \\
& 635 & 25894 & 47.26 & 41.29 & 37.35 & -0.49 & 513.67 & 2.36 & 5.14 & 18.29 & 37.35 & 64.34 & 122.67 \\
\hline \multirow{2}{*}{$\sigma_{\text {bsp }}$} & 525 & 25974 & 4.67 & 3.38 & 3.63 & -0.50 & 60.56 & 2.12 & 0.26 & 1.77 & 3.63 & 5.87 & 10.06 \\
& 450 & 25951 & 5.46 & 4.23 & 4.70 & -0.50 & 58.86 & 2.08 & 0.57 & 2.40 & 4.70 & 7.51 & 12.86 \\
\hline \multirow{6}{*}{ g } & 635 & 23963 & 0.54 & 0.10 & 0.54 & -0.97 & 0.97 & -3.34 & 0.40 & 0.50 & 0.54 & 0.59 & 0.67 \\
& 525 & 24503 & 0.59 & 0.06 & 0.59 & -0.46 & 0.90 & -1.39 & 0.49 & 0.56 & 0.59 & 0.62 & 0.68 \\
& 450 & 25038 & 0.60 & 0.07 & 0.61 & -0.94 & 0.88 & -2.30 & 0.49 & 0.58 & 0.61 & 0.64 & 0.68 \\
\hline \multirow{2}{*}{$\sigma_{\text {ap }}$} & 470 & 21580 & 7.66 & 6.50 & 6.04 & -0.25 & 94.05 & 2.48 & 1.06 & 3.12 & 6.04 & 10.35 & 19.37 \\
& 880 & 21567 & 3.51 & 2.99 & 2.73 & -0.21 & 31.43 & 2.10 & 0.44 & 1.37 & 2.73 & 4.81 & 9.02 \\
\hline \multirow{2}{*}{ SAE } & $450-635$ & 27959 & 1.38 & 0.79 & 1.42 & -2.45 & 5.98 & 0.14 & 0.06 & 1.01 & 1.42 & 1.76 & 2.48 \\
\hline AAE & $370-950$ & 21390 & 1.30 & 0.30 & 1.27 & -1.86 & 5.84 & 0.69 & 0.91 & 1.14 & 1.27 & 1.44 & 1.75 \\
\hline \multirow{2}{*}{ SSA } & 470 & 13585 & 0.83 & 0.07 & 0.84 & 0.12 & 0.98 & -1.69 & 0.70 & 0.80 & 0.84 & 0.88 & 0.91 \\
& 880 & 13575 & 0.80 & 0.12 & 0.83 & 0.05 & 1.00 & -1.71 & 0.57 & 0.75 & 0.83 & 0.89 & 0.95 \\
\hline PM & - & 35354 & 16.23 & 11.08 & 14.15 & 0.15 & 236.51 & 2.24 & 3.49 & 8.14 & 14.15 & 22.01 & 35.22 \\
\hline
\end{tabular}




\begin{tabular}{|c|c|c|c|c|c|c|c|c|c|c|c|c|c|}
\hline \multirow[t]{2}{*}{ MSA } & \multirow[t]{2}{*}{$\lambda$} & \multirow[t]{2}{*}{ Counts } & \multirow[t]{2}{*}{ Mean } & \multirow[t]{2}{*}{ SD } & \multirow[t]{2}{*}{ Median } & \multirow[t]{2}{*}{ Min } & \multirow[t]{2}{*}{ Max } & \multirow[t]{2}{*}{ Skewness } & \multicolumn{5}{|c|}{ Percentiles } \\
\hline & & & & & & & & & 5 & 25 & 50 & 75 & 95 \\
\hline \multirow{3}{*}{$\sigma_{\mathrm{sp}}$} & 635 & 21708 & 16.73 & 19.28 & 9.37 & -0.50 & 307.33 & 2.05 & 0.22 & 2.77 & 9.37 & 25.19 & 54.79 \\
\hline & 525 & 21790 & 22.12 & 25.09 & 12.44 & -0.50 & 277.46 & 1.85 & 0.40 & 3.54 & 12.44 & 33.46 & 71.78 \\
\hline & 450 & 21792 & 28.11 & 31.50 & 16.06 & -0.50 & 376.38 & 1.80 & 0.59 & 4.53 & 16.06 & 42.70 & 91.38 \\
\hline \multirow{3}{*}{$\sigma_{\mathrm{bsp}}$} & 635 & 21728 & 2.29 & 2.51 & 1.38 & -0.50 & 30.59 & 1.52 & -0.13 & 0.35 & 1.38 & 3.68 & 7.14 \\
\hline & 525 & 21757 & 2.69 & 2.91 & 1.60 & -0.50 & 36.04 & 1.45 & -0.09 & 0.41 & 1.60 & 4.30 & 8.36 \\
\hline & 450 & 21542 & 3.19 & 3.43 & 1.94 & -0.50 & 42.44 & 1.42 & -0.10 & 0.46 & 1.94 & 5.16 & 9.82 \\
\hline \multirow{3}{*}{$\mathrm{g}$} & 635 & 18287 & 0.52 & 0.17 & 0.54 & -1.00 & 0.94 & -3.10 & 0.25 & 0.48 & 0.54 & 0.60 & 0.71 \\
\hline & 525 & 18657 & 0.57 & 0.14 & 0.59 & -1.00 & 0.94 & -3.53 & 0.35 & 0.54 & 0.59 & 0.63 & 0.73 \\
\hline & 450 & 18644 & 0.60 & 0.14 & 0.62 & -1.00 & 0.94 & -3.63 & 0.38 & 0.57 & 0.62 & 0.66 & 0.77 \\
\hline \multirow{2}{*}{$\sigma_{\mathrm{ap}}$} & 470 & 9913 & 3.57 & 3.95 & 2.03 & -0.24 & 70.52 & 2.53 & 0.13 & 0.65 & 2.03 & 5.64 & 10.50 \\
\hline & 880 & 9915 & 1.59 & 1.71 & 0.89 & -0.16 & 23.03 & 1.79 & 0.06 & 0.29 & 0.89 & 2.57 & 4.81 \\
\hline SAE & $450-635$ & 20189 & 1.58 & 0.83 & 1.64 & -1.95 & 6.00 & -0.04 & 0.08 & 1.24 & 1.64 & 1.95 & 2.82 \\
\hline AAE & $370-950$ & 9625 & 1.36 & 0.27 & 1.32 & -0.90 & 4.80 & 2.02 & 1.05 & 1.21 & 1.32 & 1.47 & 1.76 \\
\hline \multirow{2}{*}{ SSA } & 470 & 7146 & 0.85 & 0.08 & 0.87 & 0.21 & 1.00 & -2.16 & 0.69 & 0.82 & 0.87 & 0.90 & 0.93 \\
\hline & 880 & 7134 & 0.82 & 0.13 & 0.85 & 0.04 & 1.00 & -2.29 & 0.57 & 0.79 & 0.85 & 0.89 & 0.95 \\
\hline $\mathrm{PM}_{10}$ & - & 18782 & 11.32 & 9.93 & 8.46 & 0.10 & 153.62 & 2.60 & 1.36 & 4.84 & 8.46 & 15.54 & 29.51 \\
\hline
\end{tabular}




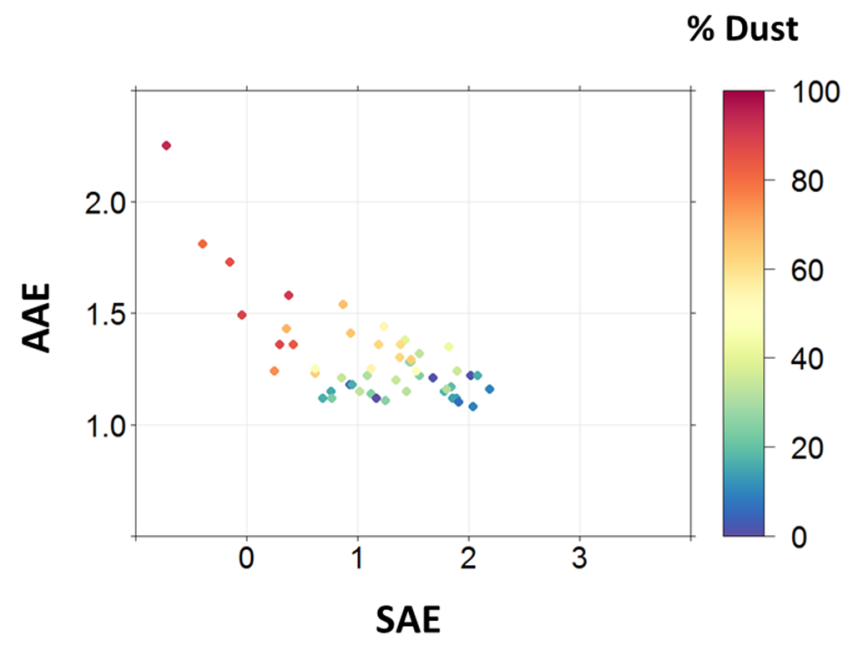

Figure S1. Ångström matrix (AAE vs. SAE weighted by \% dust in PM10) during Saharan dust events at MSY (daily base). 
a)

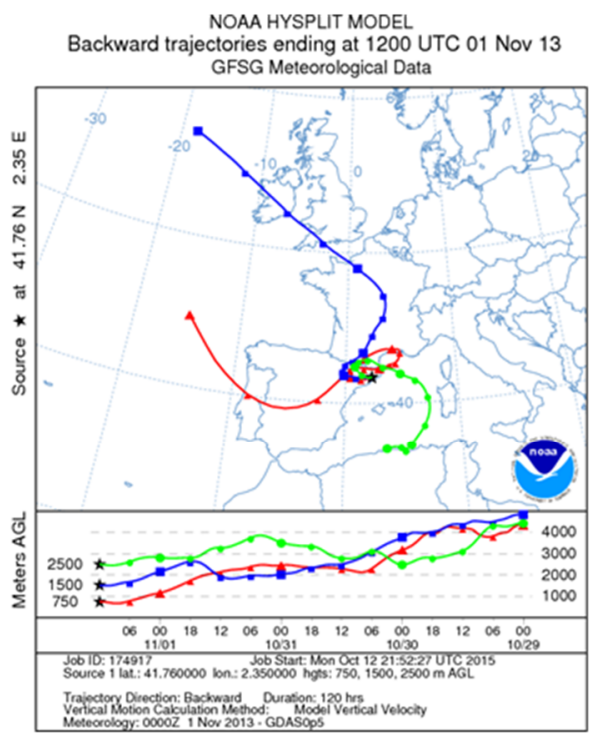

b)

NOAA HYSPLIT MODEL
Backward trajectories ending at 1200 UTC 28 Oct 13 GFSG Meteorological Data

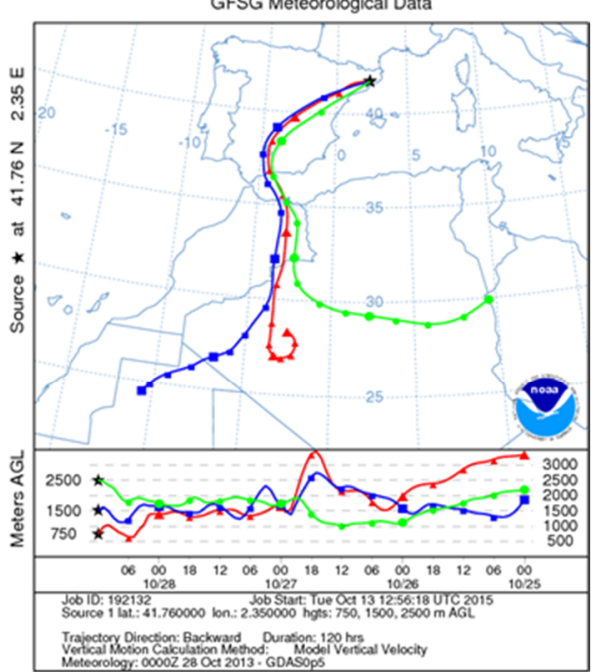

c)

BSC-DREAM8b v2.0 Dust Low Level Conc. $\left(\mu \mathrm{g} / \mathrm{m}^{3}\right)$ 00h forecast for 12UTC 28 Oct 2013

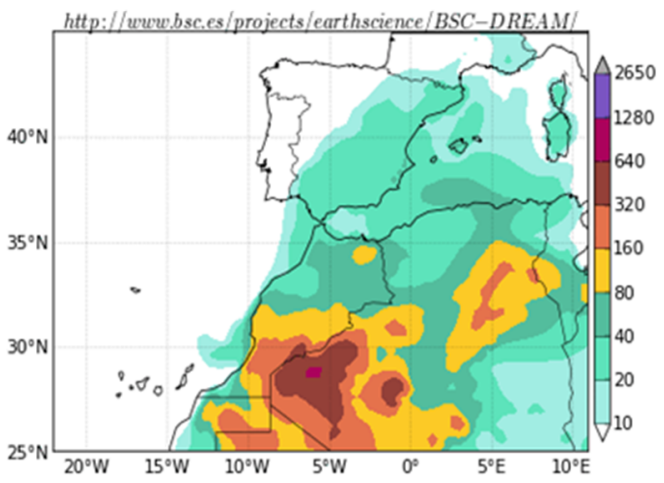

20 Figure S2. Backward trajectories corresponding to (a) regional episode (01/11/2013) and (b) Saharan dust event (28/10/2013) atmospheric scenarios at MSY. (c) Dust surface concentration at MSY from the Dream model corresponding to a Saharan dust event (28/10/2013). 
a)

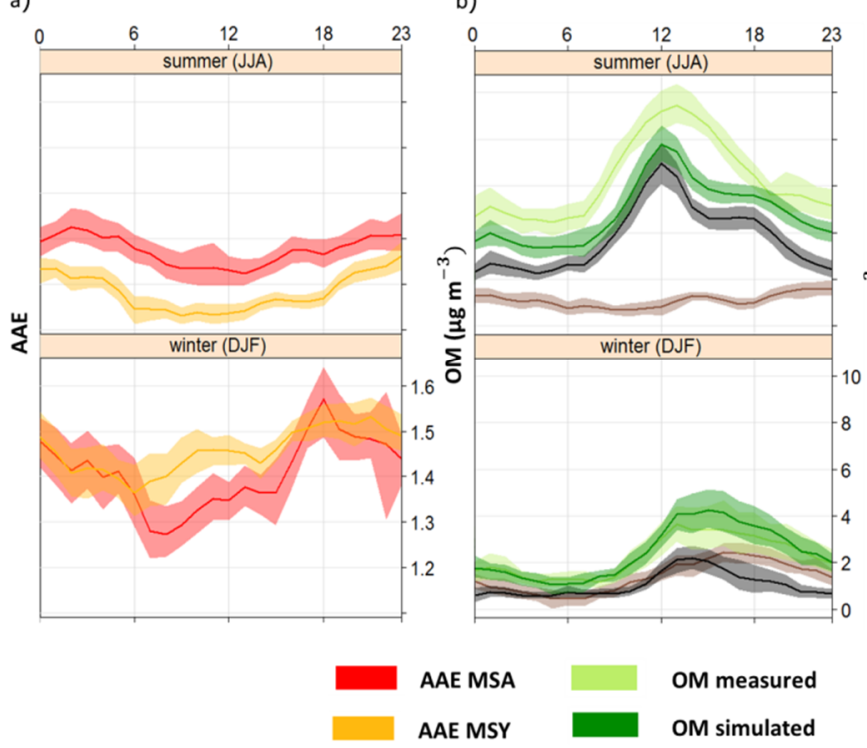

MSY

c)

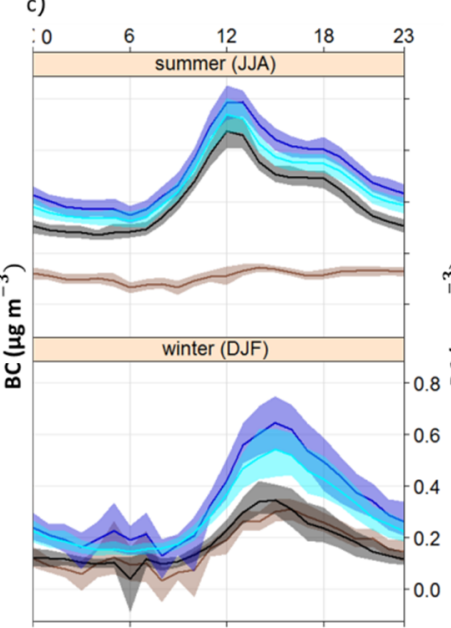

BC measured

BC simulated
MSA

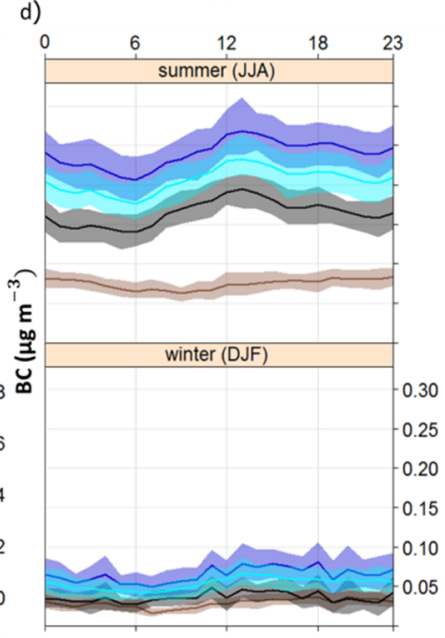

BCff, OMff

BCbb, OMbb

Figure S3. Summer and winter daily cycles of: (a) AAE at MSY and MSA, (b) measured OM and simulated OM as the sum of OMff and OMbb contributions at MSY, measured BC and simulated BC as the sum of BCff and BCbb contributions at (c) MSY and (d) MSA. Averages were calculated from hourly base. 